\title{
Corrigendum: Persisting positron emission tomography lesion activity and Mycobacterium tuberculosis mRNA after tuberculosis cure
}

Stephanus T Malherbe, Shubhada Shenai, Katharina Ronacher, Andre G Loxton, Gregory Dolganov, Magdalena Kriel, Tran Van, Ray Y Chen, James Warwick, Laura E Via, Taeksun Song, Myungsun Lee, Gary Schoolnik, Gerard Tromp, David Alland, Clifton E Barry III, Jill Winter, Gerhard Walzl, the Catalysis TB-Biomarker Consortium

Nat. Med. 22, 1094-1100 (2016); published online 5 September 2016; corrected after print 19 October 2016; corrected after print 10 November 2017

In the version of this article initially published, two authors in the Catalysis TB-Biomarker Consortium were incorrectly identified as Lani Theart and Coenie Kogelenberg. The authors' names are Lani Thiart and Coenie Koegelenberg. Also, in Figure 3c, the three patients who initiated TB retreatment after EOT + 1y had 'mixed' response patterns at EOT + 1y, as is written in the text, and not 'resolved' response patterns, as is shown in the original version of the figure. The errors have been corrected in the HTML and PDF versions of the article.

\section{Corrigendum: ADAM10-mediated ephrin-B2 shedding promotes myofibroblast activation and organ fibrosis}

David Lagares, Parisa Ghassemi-Kakroodi, Caroline Tremblay, Alba Santos, Clemens K Probst, Alicia Franklin, Daniela M Santos, Paula Grasberger, Neil Ahluwalia, Sydney B Montesi, Barry S Shea, Katharine E Black, Rachel Knipe, Meryem Blati, Murray Baron, Brian Wu, Hassan Fahmi, Rajiv Gandhi, Annie Pardo, Moisés Selman, Jiangping Wu, Jean-Pierre Pelletier, Johanne Martel-Pelletier, Andrew M Tager \& Mohit Kapoor

Nat. Med.; doi:10.1038/nm.4419; corrected online 20 November 2017

In the version of this article initially published online, the positions of the colored boxes in the key of Figure $5 \mathrm{f}$ were inverted. The treatment group is represented by the red line of the graph and the control group by the blue line. The error has been corrected in the print, PDF and HTML versions of this article. 